
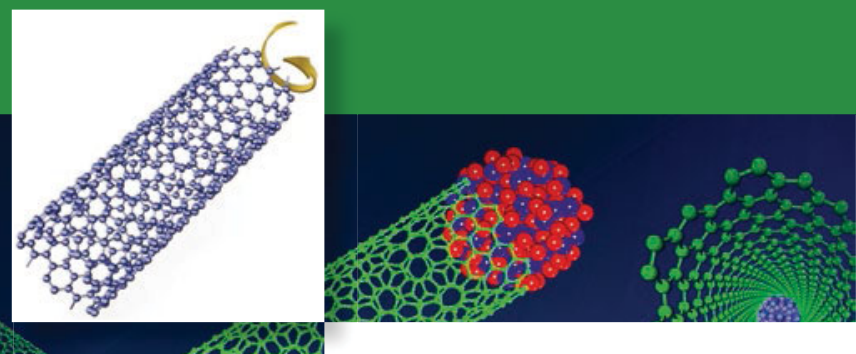

\title{
Growth of nanocarbons by catalysis and their applications
}

\section{Peter A. Thrower and Hui-Ming Cheng, Guest Editors}

\begin{abstract}
Nanocarbons, such as carbon nanotubes and graphene, have had a remarkable history and impact on current applications. We briefly review the genesis and development of nanocarbons over the last 50 years, referencing key articles, including the role of catalysts in their formation. This issue focuses on the formation mechanisms and controlled growth of carbon nanotubes and graphene on substrates through catalytic processes. The five contributions in this issue review the mechanisms and theory of catalytic growth of nanocarbons (carbon nanotube forests, superaligned arrays, single-wall carbon nanotubes), the growth of large-quantity, high-quality graphene on metal substrates, and the unique and excellent properties for current and potential commercial applications.
\end{abstract}

\section{Introduction}

It may seem strange to some readers that the idea of nanocarbons was basically nonexistent when one of us (P.T.) started work on carbon materials at the United Kingdom Atomic Energy Research Establishment, Harwell, in 1960. The materials we worked on then were macroscopic in scale, and the major funding for carbon research worldwide was from atomic-energy agencies who were interested in understanding the behavior of graphite blocks in nuclear reactors, where they were mostly used as a moderator for the highenergy neutrons produced during the fission of uranium atoms. When Thrower left Harwell in 1969 and went to work at The Pennsylvania State University, there was research on the new microcarbon, carbon fibers, conducted in his Harwell group, but it was classified as secret. The identification and systematic production of carbon nanotubes was still more than 20 years away. This does not mean that nanocarbons did not exist in those days. We simply did not have ways to separate, identify, and investigate them. R.T.K. Baker, from Harwell, for instance, was investigating fibrous carbon that sometimes formed in a graphite-moderated gas-cooled nuclear reactor, undoubtedly the result of catalytic processes. ${ }^{1}$ There is no doubt that these contained carbon nanotubes and metallic particles, but the nanotubes could not be fully identified.
In those days a "high-resolution" transmission electron microscope (TEM) could resolve only $\sim 1.2 \mathrm{~nm}$ on a good day, and it would be a few years before the atomic layers in graphite (graphene layers) were first resolved. In 1968, Heidenreich, Hess, and Ban ${ }^{2}$ used a customized TEM installation to accomplish this goal. The word "graphene" to describe a single layer of carbon atoms in graphite did not appear in the literature until 1987, although we can argue that the material has been used for centuries. When the ancient Greeks were writing with crude pencils, they had no idea they were sliding graphene sheets from graphite onto paper.

\section{Carbon nanotubes}

Carbon nanotubes were reported in 1991 by Iijima, whose Nature paper ${ }^{3}$ remains one of the most cited ones in the scientific literature ( $>44,000$ according to Google Scholar). It soon became obvious that although carbon nanotubes had a very high tensile strength, measuring their other properties was quite complex. Although a single-wall carbon nanotube (SWCNT) could be simply thought of as being formed by "rolling" a graphene sheet into a cylinder, there are many ways of doing this. As shown in Figure 1, the graphene can be rolled such that the tube axis is either parallel to or perpendicular to a carbon-carbon bond and there could also be an offset in the join, producing what is known as chirality. 


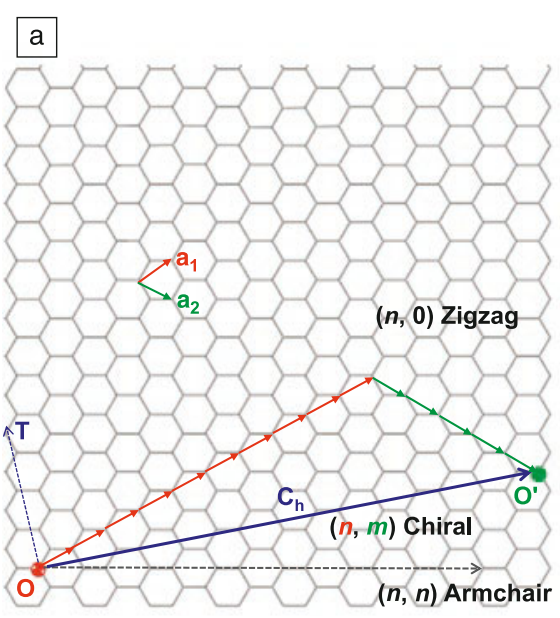

$\mathrm{b}$

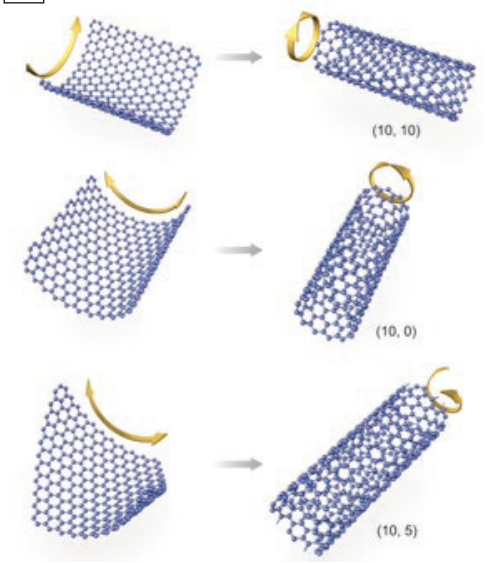

Figure 1. Diagram of the nanocarbons discussed in this issue. (a) A graphene sheet showing the two lattice vectors $\mathbf{a}_{1}$ and $\mathbf{a}_{2}$. Single-wall carbon nanotubes (SWCNTs) are produced by rolling up a graphene sheet around an axis $n a_{1}+m a_{2}$. When $m=0$, a zigzag nanotube is produced, and when $n=m$, the nanotube is armchair. All other situations result in a chiral nanotube where $(n, m)$ is known as the chirality index. The three SWCNTs shown in (b) are $(10,10)$ armchair, $(10,0)$ zigzag, and $(10,5)$ chiral, formed by rolling up a graphene sheet in these different ways. In (a), $\mathrm{O}$ and $\mathrm{O}^{\prime}$ represent the positions of the two carbon atoms in the graphene lattice, which coincide when the graphene is rolled into a $(10,5)$ chiral SWCNT. T, which is perpendicular to $\mathbf{O O}^{\prime}$, denotes the radial direction of the SWCNT. $\mathbf{C}_{h}$ is the lattice vector of the SWCNT, and the length of $\mathbf{C}_{h}$ is the circumference of the SWCNT. The red, green, and blue arrows respectively represent the $\mathbf{a}_{\mathbf{1}}$ and $\mathbf{a}_{\mathbf{2}}$ vectors, and the OO' vector. Courtesy of F. Li and B.L. Liu.

layers of the graphite structure by reduction of graphite oxide." ${ }^{5}$ His suggestion for producing single layers by the reduction of graphite oxide turned out to be correct and important. The corresponding paper included a table of estimated layer thicknesses, with a few in the range $0.3-0.6 \mathrm{~nm}$. The following year, he published a paper titled "Dünnste Kohlenstoff-Folien" (Thinnest carbon films), ${ }^{6}$ in which these data were explored more fully, and it was suggested that one of the photographs might be that of a single layer of carbon. It was not until 25 years later, in 1987, that the term "graphene" was introduced ${ }^{7,8}$ in relation to graphite intercalation compounds. In a 2010 essay, Boehm attempted to clarify some of the early work on the topic and indicated that "the term (graphene) was recommended by the relevant IUPAC (International Union of Pure and Applied Chemistry) commission on the suggestion of Eberhard Stumpp (Technische Universität Clausthal) and a subcommittee of the Working Carbon Group of the German Ceramic Society to enable characterization of the properties of single two-dimensional layers which exist independently of neighboring carbon layers." 9

Intercalation compounds were a hot topic

These structural differences mean that while carbon nanotubes can be electrically conducting (metallic), they can also be semiconducting, with a bandgap that depends on the rolling and joining process. Added to this was another complexity-it was possible to have concentric nanotubes, giving rise to double-wall, triple-wall, and multiwall varieties. It is now clear that the structure of the nanotube produced is related to the catalyst particle used to initiate its growth, and the production of nanotubes, all with the same controlled properties, remains an important research endeavor.

\section{The rise of graphene}

In 1960, several researchers were preparing thin graphite specimens for electron microscope studies from single-crystal graphite by its repeated cleavage using Scotch Tape (USA), or Sellotape (UK), depending on where you were working. One well remembers holding the tape up to light and seeing transparent regions that one would then try to isolate, and wondering how many layers they contained. The only guide to answering this question was an investigation of light transmission through amorphous carbon films as a function of thickness, ${ }^{4}$ but all that did was to indicate that the thickness had to be less than $100 \mathrm{~nm}$. We simply did not have the means to measure such small thicknesses. There is no doubt that we occasionally produced single layers, but defining them and investigating their properties would not come until several years later.

In 1961, H.-P. Boehm suggested at the Fifth Biennial US Carbon Conference that "it should be possible to obtain single in carbon materials science in the $1980 \mathrm{~s} .{ }^{10}$ They were the focus of the majority of papers submitted to the journal Carbon, and the subject dominated the annual Carbon conferences at that time. Research promised materials (incorrectly as it turned out) whose electrical conductivity would far exceed that of copper and led to the development of the lithium-ion batteries that are now so prevalent. It was in the intercalation materials that the term graphene was first used, because the intercalating species separated the graphene layers in graphite.

Geim and Novoselov isolated and characterized graphene in 2004 at the University of Manchester, UK. ${ }^{11}$ This work resulted in their being awarded the Nobel Prize in Physics in 2010 "for groundbreaking experiments regarding the twodimensional material graphene." Since that time, there have been thousands of research papers on graphene with one major topic being its growth on catalytic substrates. ${ }^{12}$

\section{Nanocarbon materials formation}

It is clear that the formation mechanism and controlled growth of carbon nanotubes and graphene on substrates are largely determined by catalytic processes. The subject of graphene formation is relatively simple though there are other forms of the material, for example, bilayer or trilayer graphene, ${ }^{13}$ whereas carbon nanotubes are multiform, with differences in the number of tube walls, the chirality of the graphene sheets in the walls, and the alignment directions (vertical or horizontal), ${ }^{14}$ resulting in many different properties (Figure 1). Much current research, both experimental and theoretical, 
is concerned with the production of nanotubes of a single form (e.g., semiconducting or even single chirality), ${ }^{14}$ and this has involved the investigation of a large number of catalysts such as $\mathrm{Co}-\mathrm{Mo}, \mathrm{W}_{6} \mathrm{Co}_{7}$, and $\mathrm{Mo}_{2} \mathrm{C}$. A major aim in graphene research is the production of large single-crystal areas of the material, and this requires the availability of large single-crystal catalytic substrates. ${ }^{15,16}$

The articles in this issue consider theoretical predictions, elucidation of the growth mechanisms of nanocarbons on metal catalysts, the commercial applications of carbon nanotubes, and graphene grown on catalysts in large quantities with high quality.

\section{In this issue}

In their article in this issue, Penev et al. ${ }^{17}$ introduce the mechanisms and theory of the catalytic growth of nanocarbons, with the aim of solving the problems of the well-controlled production of these quintessential nanomaterials. They highlight how experimental discoveries and theoretical approaches have evolved hand-in-hand, resulting in a successful understanding or in unresolved issues, and discuss possible parallels and distinctions between graphene and CNTs, aiming not only to offer available answers, but also to emphasize current and future challenges.

Chen et al., ${ }^{18}$ in their article, provide a general picture of two decades of research and development on the catalytic growth of CNT "forests" and superaligned arrays, a unique vertically aligned structure of CNTs. When the size and spacing of catalyst nanoparticles are well controlled on a substrate, long, aligned, and pure CNTs can be easily synthesized in simple or highly complex configurations.

The holy grail of CNT growth is fabricating singlechirality CNTs. In their article, Zhao et al. ${ }^{19}$ introduce us to catalysts used for SWCNT synthesis-from structure-controlled growth to mass production. Catalysts play essential roles in the growth of SWCNTs, in particular, with a specific chirality. Catalysts with a high melting point, a specific atomic arrangement, and reasonable activity are the rational choice for the chirality-selective growth of SWCNTs. In the bulk synthesis of SWCNTs, future attention may shift from high-yield growth to structure-controlled growth.

Similar to the catalytic growth of CNTs, the synthesis of graphene by chemical vapor deposition (CVD) occurs on catalyst substrates. In their article, Qing et al. ${ }^{20}$ focus on catalytic substrates, especially metals, for graphene growth using CVD. They discuss graphene growth mechanisms and kinetics, control of the number of graphene layers and stacking order, engineering of large-area graphene single crystals, and low-temperature growth. Finally, they highlight the challenges and prospects in the field.

One of the major aims of the research and development of new materials is their commercial use. Nanocarbons have unique and excellent properties, and consequently, various applications, such as electronics, optoelectronics, energy storage, composites, and sensors are expected and promised. These are highlighted in the Hou et al..$^{21}$ article in this issue.
The important milestones in these applications are discussed with special emphasis on the latest advances and remaining challenges. The article also compares the key characteristics of nanocarbons and the advantages of CNTs over graphene, and vice versa, for different uses.

We hope this issue will provide insights and provoke discussions into the ways catalysts influence the production of these carbon nanomaterials and how we may expect to see them used in the not-too-distant future.

\section{Dedication-Millie Dresselhaus}

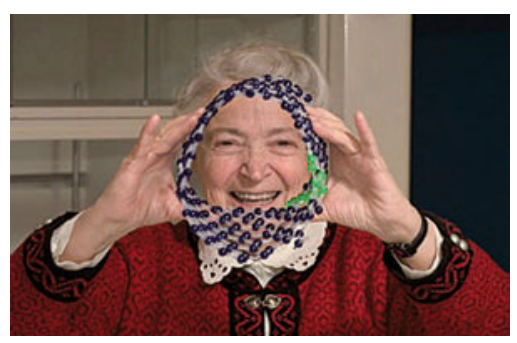

Millie Dresselhaus peering through a model of a single-wall carbon nanotube. Photo courtesy of Ed Quinn. Reproduced with permission from MIT.

The concept of this issue of MRS Bulletin originated in early February 2017, when Professor Mildred (Millie) Dresselhaus of the Massachusetts Institute of Technology and P. Thrower were invited by MRS Bulletin editors to compile and edit a special issue on the role of catalysts in nanocarbon production. Millie failed to respond, and it was a week later that we learned the shocking news of her death on February 20. P.T.'s immediate reaction was to dedicate the issue to her memory and to invite H.-M. Cheng, one of Millie's former visiting scholars, to collaborate on the project. Some people have rightly referred to Millie as "The Queen of Carbon Science." Her research activities, especially concerning nanocarbons, remained tremendous until the end of her life. She never retired! Millie's passing is a great loss to the carbon research community and materials science as a whole. We are proud and humbled to be able to dedicate this issue of MRS Bulletin in her memory.

\section{References}

1. R.T.K. Baker, Carbon 27, 315 (1989).

2. R.D. Heidenreich, W.M. Hess, L.L. Ban, J. Appl. Crystallogr. 1, 1 (1968).

3. S. lijima, Nature 354, 56 (1991).

4. A. Cosslett, V.E. Cosslett, Br. J. Appl. Phys. 8, 374 (1957)

5. H.P. Boehm, A. Clauss, G. Fischer, U. Hoffmann, "Surface Properties of Extremely Thin Graphite Lamellae," Proc. 5th Conf. Carbon (Pergamon Press, Oxford, UK, 1961), pp. 73-80.

6. H.P. Boehm, A. Clauss, G.O. Fischer, U. Hoffmann, Z. Naturforsch. B Chem. Sci. 17b, 150 (1962).

7. S. Mouras, A. Hamm, D. Djurado, J.-C. Couseins, Rev. Chim. Minér. 24, 572 (1987).

8. H.P. Boehm, R. Setton, E. Stumpp, Pure Appl. Chem. 66, 1893 (1994).

9. H.P. Boehm, Angew. Chem. Int. Ed. 49, 9332 (2010).

10. M. Dresselhaus, G. Dresselhaus, Adv. Phys. 30, 139 (1981).

11. K.S. Novoselov, A.K. Geim, S.V. Morosov, G. Jiang, Y. Zhang, S.V. Dubonos,

I.V. Grigorieva, A.A. Firsov, Science 306, 666 (2004)

12. Y. Zhang, L. Zhang, C. Zhou, Acc. Chem. Res. 46, 2329 (2013)

13. E. McCann, M. Koshino, Rep. Prog. Phys. 76, 056503 (2013). 
14. F. Yang, X. Wang, M. Li, X. Liu, X. Zhao, D. Zhang, Y. Zhang, J. Yang, Y. Li, Acc. Chem. Res. 49, 606 (2016).

15. X. Xua, Z. Zhang, J. Dong, D. Yi, J. Niu, M. Wu, L. Lin, R. Yin, M. Li, J. Zhou,

S. Wang, J. Sun, X. Duan, P. Gao, Y. Jiang, X. Wu, H. Peng, R.S. Ruoff, Z.F. Liu,

D. Yu, E. Wang, F. Ding, K. Liu, Sci. Bull. 62, 1074 (2017).

16. H.M. Cheng, Sci. Bull. 62, 1039 (2017).

17. E.S. Penev, F. Ding, B.I. Yakobson, MRS Bull. 42 (11), 794 (2017).

18. G. Chen, D.N. Futaba, K. Hata, MRS Bull. 42 (11), 802 (2017).

19. X. Zhao, S. Zhang, Z. Zhu, J. Zhang, F. Wei, Y. Li, MRS Bull. 42 (11), 809 (2017). 20. F. Qing, C. Shen, R. Jia, L. Zhan, X. Li, MRS Bull. 42 (11), 819 (2017).

21. P.-X. Hou, J. Du, C. Liu, W. Ren, E.I. Kauppinen, H.-M. Cheng, MRS Bull. 42 (11), 825 (2017).

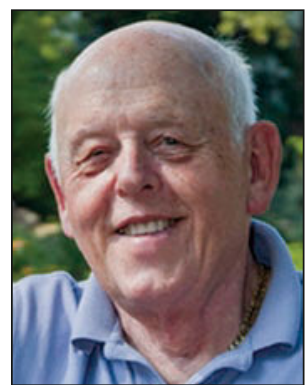

Peter A. Thrower has been retired from the Department of Materials Science and Engineering at The Pennsylvania State University (Penn State) since 1998. He graduated in 1960 from the University of Cambridge, UK, with a BA degree in physics. He was employed by the United Kingdom Atomic Energy Research Establishment at Harwell, and was awarded a PhD degree in 1969 by the University of Cambridge as a result of his Harwell research. He then joined Penn State as an associate professor. In 1983, he was appointed professor of materials science and was also appointed editor-in-chief of the journal Carbon, continuing to edit the journal until 2013. His current work focuses on assisting researchers in China and Korea by advising on and editing their scientific work for publication. Thrower can be reached by phone at +44 1692678161 or by email at peter.thrower@cantab.net.

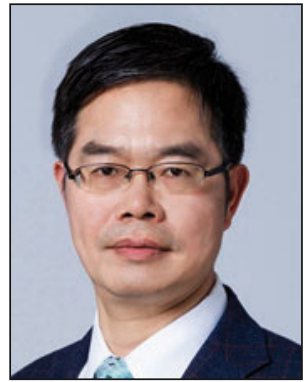

Hui-Ming Cheng is professor and director of the Advanced Carbon Research Division of Shenyang National Laboratory for Materials Science, Institute of Metal Research, Chinese Academy of Sciences, as well as the Low-Dimensional Material and Device Laboratory of the Tsinghua-Berkeley Shenzhen Institute, Tsinghua University, China. He received his BA degree in 1984 in carbon materials from Hunan University, China, and his master's degree and PhD degree in 1987 and 1992, respectively, from the Institute of Metal Research, Chinese Academy of Sciences. His research focuses on the synthesis and applications of carbon nanotubes, graphene, other lowdimensional materials, energy-storage materials, photocatalytic semiconducting materials, and bulk carbon materials. Cheng can be reached by phone at $+86-24-$ 23971788 or by email at cheng@imr.ac.cn or hmcheng@sz.tsinghua.edu.cn.

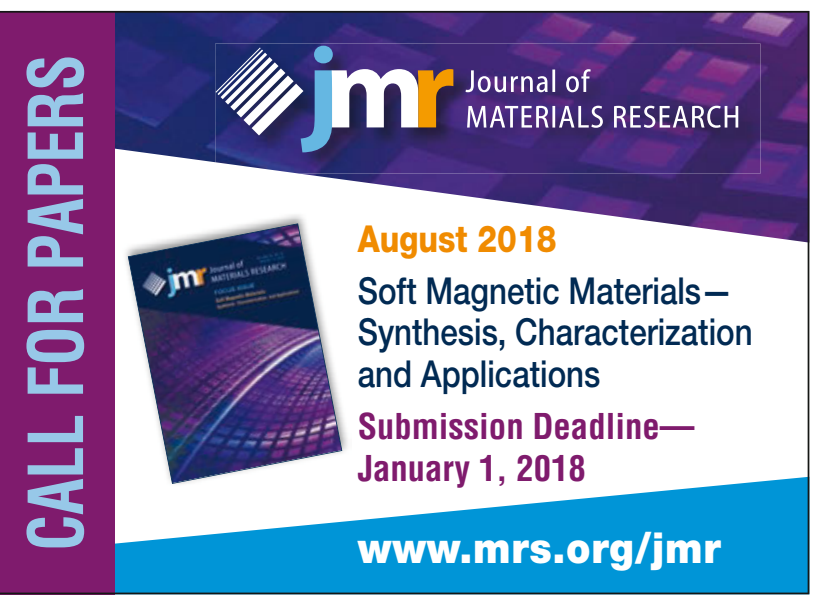

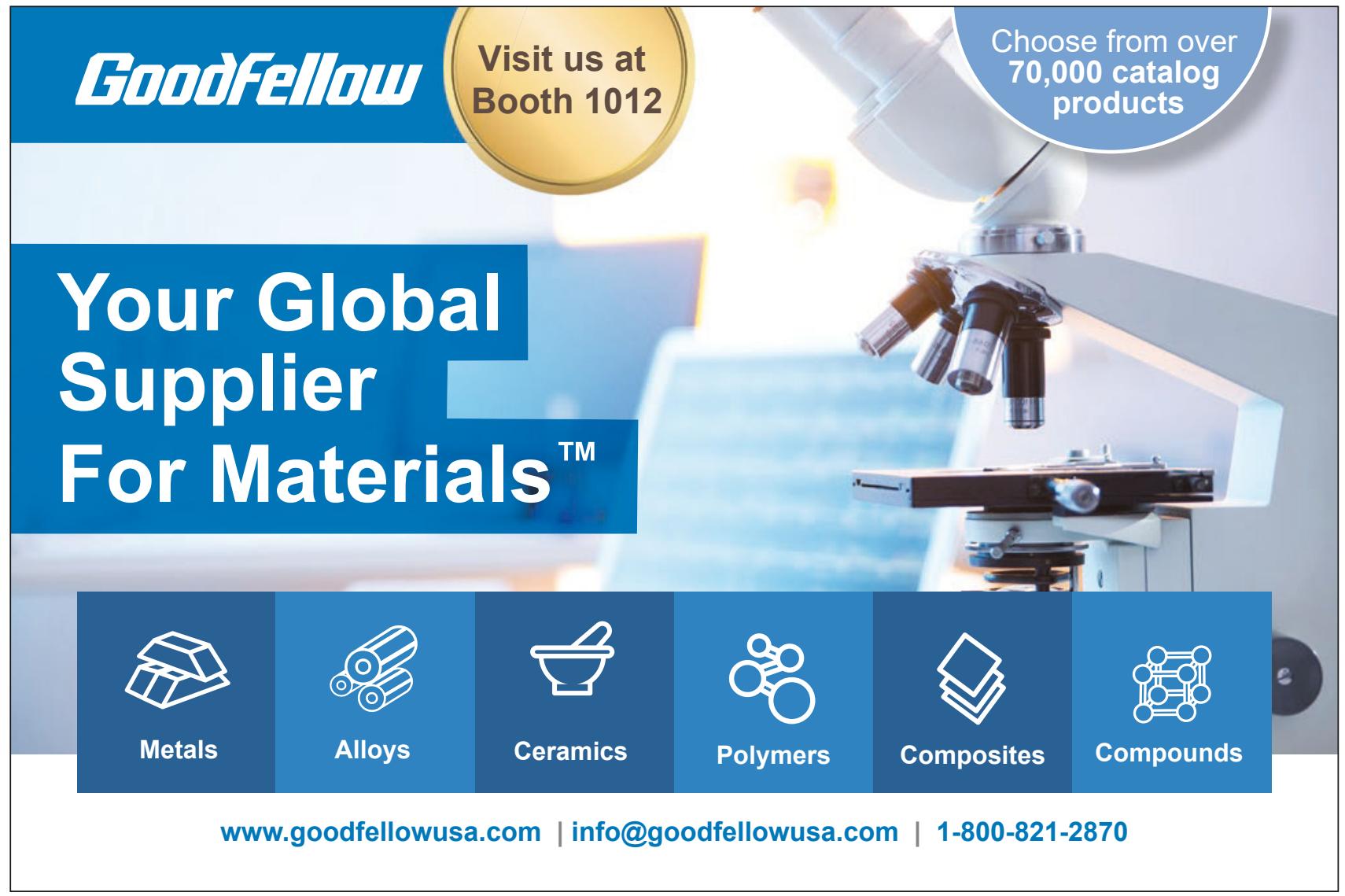

